\title{
Resolution Capabilities of Future THz Cameras
}

\author{
Juan LIZARRAGA ${ }^{1}$, Carlos DEL-RIO ${ }^{2}$ \\ ${ }^{1}$ European Space Agency, Keplerlaan 1, 2201 AZ Noordwijk, The Netherlands \\ ${ }^{2}$ Antenna Group, Public University of Navarra, Campus Arrosadía s/n, 31006, Pamplona, Spain \\ juan.lizarraga.cubillos@esa.int, carlos@unavarra.es
}

\begin{abstract}
THz technology for developing imaging systems has recently aroused great interest, mainly due to the large number of applications in which these frequencies can be used: security, vision in hard environments, etc.

In this paper we propose a method that reduces significantly the number of detectors needed for achieving certain resolution by means of diffraction that paradoxically is its main limiting factor in current imaging devices. The method uses diffraction as a way of achieving the advantages of the spatial diversity (information spread over a set of detectors) giving also the possibility to increase the resolution of the obtained images interpolating samples between detectors thanks to the slow variation function created by the diffraction phenomena.
\end{abstract}

\section{Keywords}

Terahertz cameras, resolution improvement, CCD.

\section{Introduction}

$\mathrm{THz}$ technology despite having a great potential still faces limitations that hinder its development. The most important one is the difficulty to produce detectors in large numbers at these frequencies. This fact poses serious constrains to the possibilities of the imaging systems that use them. Many times, the system has to include some kind of moving mirrors in order to scan the entire vision field. These limitations boost research towards finding alternative systems and techniques that allow us to overcome the shortcomings current technology has.

When talking about vision systems it is mandatory to mention its main detection device in the optical frequency band: the CCD (Charge-Coupled Device). The CCDs are the paradigm of individual non-cooperative detection. Although the elements of a CCD make up an array of sensors, they do not work as one antenna-wise (cooperation among elements), but each one is exclusively responsible for detecting the information that comes to it. This conception, which can be extrapolated to lower frequencies, limits the maximum achievable resolution, the robustness and the complexity of the system. The former is limited by the size of the elements, the spacing among them and the total number of detectors in the array. The robustness is limited to that of a single element since the failure of any of them means the loss of the information the damaged one was supposed to receive. Finally, the complexity increases due to the fact that individual detection itself requires a large number of detectors along with the control elements associated to each of them.

Nowadays it is technologically affordable to take this non-cooperative approach at optical frequencies since components that work in this band are simple and can be produced massively, therefore cheaply, in a highly mastered technology such as silicon or CMOS. On the other hand we have the low $\mathrm{THz}$ band which lies between the optical and the microwave domain. Neither optical technology nor the microwave one provide the necessary tools for developing satisfactory solutions at these frequencies. Current devices working in this band rely on complex detectors that need heterodyne receivers to work in an intermediate frequency that present RF technology can handle. This complexity seriously limits the amount of detectors that can be used in an array.

Nature has found its way to get round some of these weaknesses, achieving more robust and less complex systems. One of the most representative exponents of this success is human eye. Even though it is not fully understood how it works, it is certainly true, and so demonstrates the evidence, that its acuity is beyond the theoretical limit it is supposed to have. Classical approaches (usually based on ray theory) fail to explain this fact and further research has yet to be made. This work ascribes this capability to a combination of spatial diversity of the information and cooperative detection, and aims to explore its usage in imaging systems.

We have developed a method that uses spatial diversity along with cooperative detection to get round the shortcomings presented above. It improves the system's robustness while lowering its complexity. The way of applying it to the CCD case is by forcing each beam of light to scatter (using a pinhole for example) so that it illuminates not a spot (single detector) but a region. By doing so the information is spread among several detectors rather than being received by a single one (spatial diversity). This represents a step forward in terms of robustness: if one or several detectors fail the information can still be recovered from those others receiving it. In addition, spatial scattering 
using a pinhole is a reversible linear transformation so the signal's original spatial distribution can be recovered. At this point it is important to highlight that if signal level rather than signal power detection is used, then when being reconstructed (spatially), it adds up in amplitude while the noise adds up in power (it is received in phase in all detectors), this allows a smaller SNR in each detector, therefore it can be simpler.

Furthermore, spatial diversity also offers a second possibility. Given the fact that the information is received by several detectors we can get rid of some of them and calculate their approximate value through interpolation. This technique reduces significantly the number of detectors required for determining the incoming point of the signal and its original value, thus diminishing the overall complexity of the system. Since decimating implies aliasing, the image has to be band-limited. This does not represent an important constrain as most images have the majority of their energy at low (spatial) frequencies.

\section{Diffraction}

Diffraction is a phenomenon present in every single imaging system. It is the main constrain to the maximum resolution achievable. It arises from the finite nature of any real imaging system compared with the infinitude of the incoming plane wave. This finitude produces the spatial windowing of the latter. The main effect of diffractions is transforming point sources in the landscape into blobs on the image. The shape of these blobs (also known as diffraction patterns) depends on the shape of the system's smallest aperture.

A rigorous study of such a phenomenon would include the use of Maxwell's equations given the electromagnetic nature of light. However several approximations have been made along the History, being the ones made by Arnold Sommerfeld, Jean Fresnel and Joseph von Fraunhofer the most relevant ones. All of them treat light as a scalar phenomenon neglecting the intrinsic vectorial nature of electromagnetic fields as described in Maxwell's equations. The good news is that in the microwave region of the spectrum, under certain conditions, these approximations produce very accurate results. In our case we have used the Fraunhofer approximation.

The Fraunhofer approximation gives an accurate estimation of diffraction pattern of an aperture under the following conditions:

- The dielectric medium is linear, isotropic, homogeneous, nondispersive and nonmagnetic.

- The diffracting aperture is large enough compared with the wavelength.

- The diffracting fields must not be observed too close from the aperture (far field).

The mathematical formulation assumes a diffracting aperture lying in the $(\xi, \eta)$ plane illuminated in the $z$ positive direction according to Fig. 1. The illumination consist of a monochromatic scalar field $u(P, t)=A(P) \cos (2 \pi v t$ $\phi(P))$ being $P$ any point in the $(\xi, \eta)$ plane and $t$ the time. For simplicity it can also be represented as a phasor $U(P)=A(P) e^{j \phi(P)}$. According to this, the diffraction pattern produced at the image plane $(\mathrm{U}, \mathrm{V})$, parallel to $(\xi, \eta)$, located at a distance $F$ is given by (1) which is nothing else but the Fourier transform of the field distribution in the aperture itself.

$$
U(u, v)=\frac{e^{j k F}}{j \lambda F} e^{j \frac{k}{2 F}\left(u^{2}+v^{2}\right)} \int_{-\infty}^{\infty} \int_{-\infty}^{\infty} U(\xi, \eta) e^{-j \frac{2 \pi}{\lambda \cdot F}(u \xi+v \eta)} d \xi d \eta
$$

In order to control and define the diffraction pattern, a circular diaphragm is commonly used. The intensity diffraction pattern it produces is given by (2) and (3).

$$
\begin{gathered}
I(\theta)=I_{0}\left(\frac{2 J_{1}(k a \cdot \sin (\theta))}{k a \cdot \sin (\theta)}\right)^{2}, \\
I_{0}=\frac{P_{0} \pi a^{2}}{\lambda^{2} F^{2}}
\end{gathered}
$$

where $a$ is the radius, $I_{0}$ is the maximum intensity at the centre of the disk, $J_{1}$ is the Bessel function of the first kind and order one, $\theta$ is the angle between the axis perpendicular to the aperture and centered on in and the line between the observation point and the aperture's centre, $P_{0}$ is the incident power at the aperture, $\lambda$ is the wavelength and $k=2 \pi / \lambda$ is the wave number. The resulting profile is shown in Fig. 2.

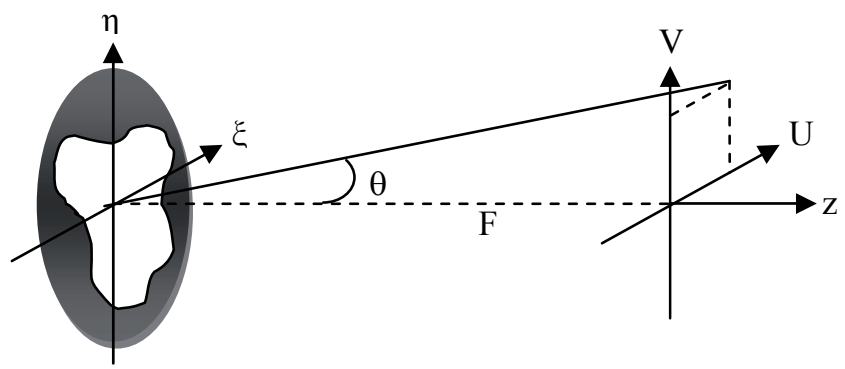

Fig. 1. Scheme of variables for the Fraunhofer approximation.

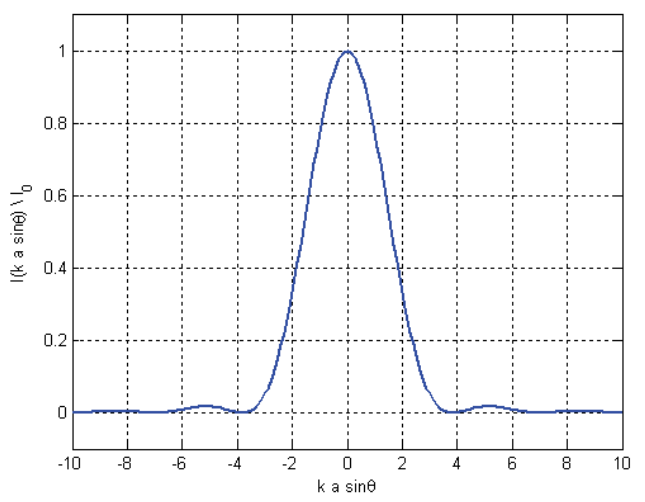

Fig. 2. Airy disk's intensity profile. 
This intensity pattern is called "Airy Disk" after G. B. Airy who first calculated it and corresponds to the intensity of the Fraunhofer diffraction pattern of the circular aperture. Its importance arises from the fact that it is used for defining the theoretical maximum resolution limit of an imaging system.

\section{Diffraction Vs. Resolution}

The question now is: how close can two Airy disks be before they stop being distinguishable? In a diffractionlimited imaging system with a circular pupil two incoherent point sources are said to be "barely resolved" when the center of the Airy intensity pattern generated by one of them falls exactly on the first zero of the Airy pattern generated by the other, Fig. 3. This criterion is known as the Rayleigh criterion.

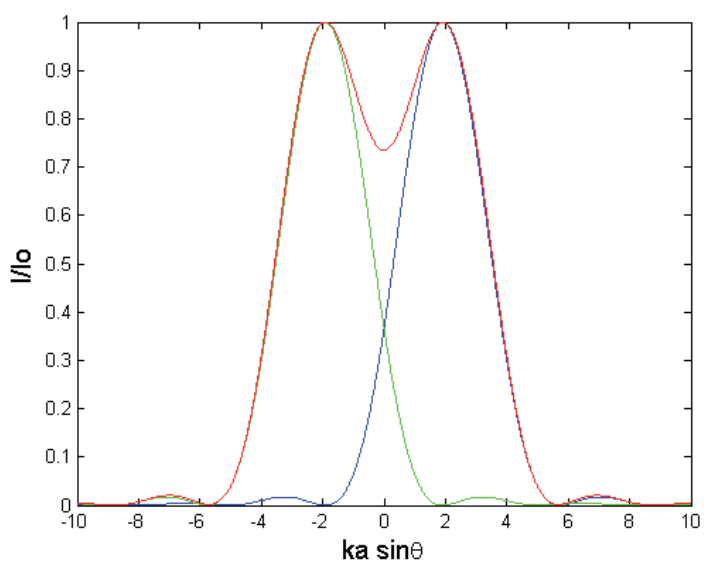

Fig. 3. The Rayleigh criterion.

The relation between this criterion and the maximum resolution achievable in a classical imaging sensor such as the CCD is simple. In a CCD the resolution is given by the size and number of sensors: the smaller (i.e. the more) the sensors are, the more resolution you get (Fig. 4). But this cannot be applied endlessly. The maximum resolution achievable will be given by the smallest sensor size which still allows the diffraction pattern to fit most of its energy within a single detector, Fig. 5. If smaller sensors are used the result is a blurry image. This is why these systems are diffraction limited.

The Rayleigh criterion establishes that the minimum distance to formally be able to distinguish two identical Airy Disk functions at the image plane is given by their radius:

$$
r=1.22 \frac{\lambda F}{2 a} .
$$

Using average dimensions of a human eye $(F=16 \mathrm{~mm}, \lambda=555 \mathrm{~nm}, \quad a=1.5 \mathrm{~mm})$ in the equation above, it yields a minimum distance on the retina of about $3.6 \mu \mathrm{m}$. Given that the diameter of a photo-detector is $1.5 \mu \mathrm{m}$, that the separation among sensors is about $0.5 \mu \mathrm{m}$ and that the Airy Disk diameter is $7.2 \mu \mathrm{m}$ two conclusions are evident: first, a single Airy Disk covers several photodetectors therefore it should create a blurry image, according to the diffraction limit for CCDs; second, the minimum detectable detail size, given by the minimum angle of resolution through this criterion, is about 50 seconds of arc.
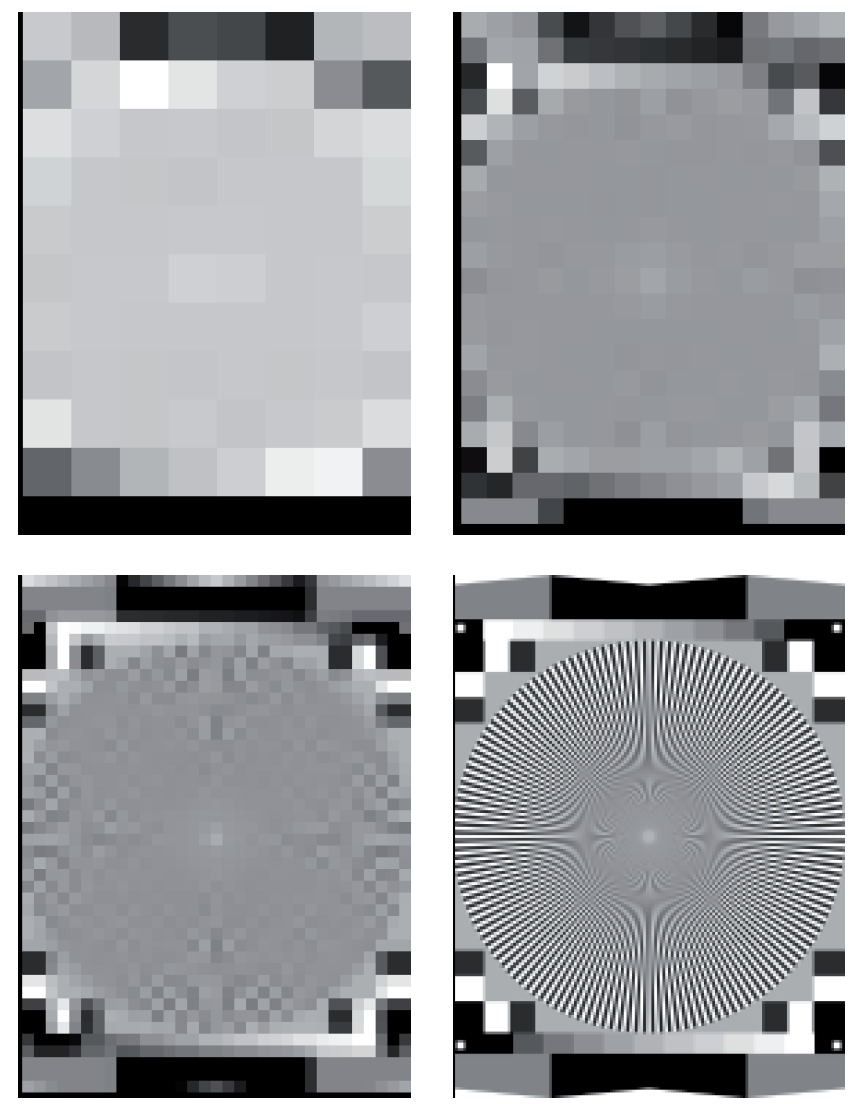

Fig. 4. Resolution improvement of the obtained image as the sensor size is reduced, being increased the number of them in a CCD.

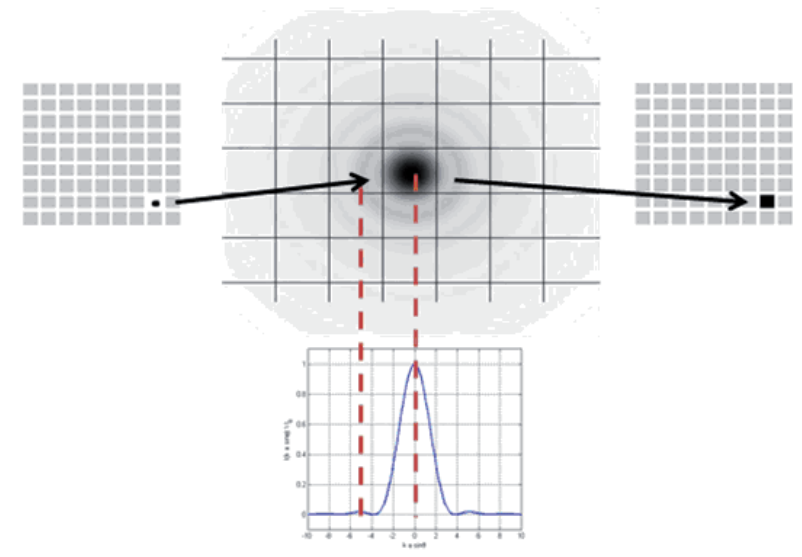

Fig. 5. Diffraction vs Resolution in a CCD.

Neither the apparent blur nor the minimum angle of resolution limits the real capabilities of the human eye, since we are able to see clear images and details much smaller than those. All this suggest that there are mechanisms -different from the CCD approach- which allow resolution beyond the diffraction limit. 


\section{Fourier Optics and Signal Detection}

Imaging systems can be studied using a powerful set of mathematical tools known as Fourier Optics. These tools, as they name suggests, use Fourier analysis and synthesis to study classical optics. They are particularly appropriate and handy for studying Fraunhofer diffraction patterns and its impact in imaging systems.

Let's call $U_{g}(u, v)$ the diffraction-free image predicted by geometrical optics. According to [5] the image produced by a diffraction-limited space-invariant imaging system will be given by (5).

$$
U_{i}(u, v)=\int_{-\infty}^{\infty} \int_{-\infty}^{\infty} h_{\lambda, F}(u-\xi, v-\eta) \cdot U_{g}(\xi, \eta) d \xi d \eta
$$

where $h_{\lambda, F}(u, v)$ is the amplitude impulse response (corresponding to the Fraunhofer diffraction pattern) of the exit pupil for a wavelength $\lambda$ at a distance $F$. This is nothing but the convolution of both functions; therefore all Fourier transform properties apply.

However actual images are not monochromatic, but polychromatic. It turns out that under narrow band conditions (as is the case of imaging systems) one can consider the amplitude impulse response to be approximately con$\operatorname{stant}\left(h_{\lambda, F}(u, v) \approx h_{F}(u, v)\right)$.

Even so, under polychromatic incoherent illumination the resulting phasor $U(u, v, t)$ is the sum of the different components at the various frequencies. As a result the different impulse responses (that we assume to be the same) interact in an uncorrelated fashion causing it to vary over time; therefore they must be added in power rather than in amplitude. When power is involved then luminous intensity plays a key role.

In Physics, intensity is the measure of the time-averaged energy flux. The need to time-average the instantaneous intensity arises from the long time it takes to the detector to integrate the incoming power compared to the reciprocal of the bandwidth. For an electromagnetic wave this measure is given by time-averaging the Poynting vector associated with it (6). Luckily Fourier analysis is still valid for incoherent imaging systems as shown in (7).

$$
\begin{gathered}
i(u, v)=\left\langle|U(u, v, t)|^{2}\right\rangle \\
i_{i}(u, v)=\int_{-\infty-\infty}^{\infty} \int_{\lambda, F}^{\infty}\left|h_{\lambda-\infty}(u-v-\eta)\right|^{2} \cdot i_{g}(\xi, \eta) d \xi d \eta
\end{gathered}
$$

The intensity profile appears at the image plane where the sensors are located, generally, in a regular matrix-like fashion.

Without loosing generality, the 1D detection process works as follows. The output level of the detector of length $X$ spanning from $a$ to $b$ (Fig. 6) using an integration time $T$ is given by (8).

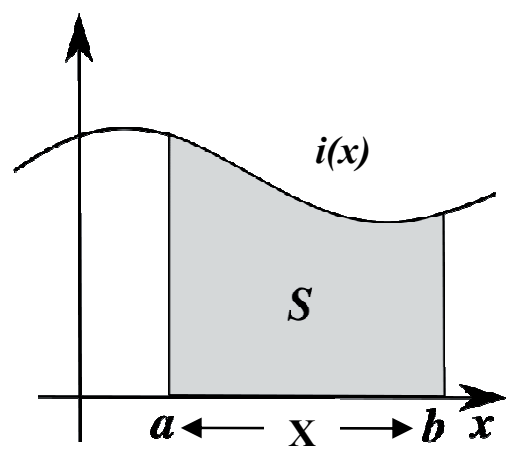

Fig. 6. Intensity profile detection.

$$
p(a, b)=T \int_{a}^{b} i(x) d x=T \cdot S .
$$

Assuming regularly-spaced equal-sized contiguous sensors, the detection process is equivalent to the linear system presented in Fig. 7.

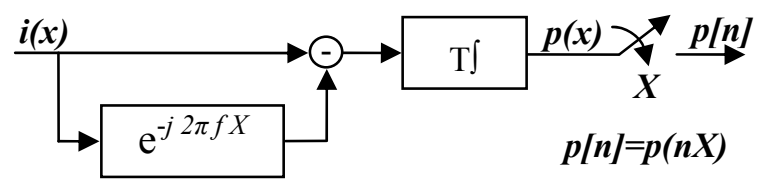

Fig. 7. Intensity profile detection equivalent linear system.

The spatial spectrum $P(f)$ of the detected light intensity profile $p(x)$ is given by (9)

$$
P(f)=T \frac{1}{\pi \cdot f} I(f) e^{-j \pi \cdot f \cdot x} \sin (\pi \cdot f \cdot X) .
$$

It is clear that the zeros introduced by the sinusoidal term destroy information. The way of addressing this issue is taking advantage of the fact that sine's period is $2 / X$ so to locate its zeros as far as possible then small values of $X$ have to be used. For such values the spectrum in (9) can be approximated by a simpler expression shown in (10)

$$
P(f) \approx T \cdot X \cdot I(f) \cdot e^{-j \pi \cdot f \cdot X} .
$$

However the smaller $X$ is the less energy is detected by each sensor. For this reason it has to be compensated by extending the exposure time $T$. In the end, a trade-off between the two quantities has to be made. The final result will depend on the relation between the sine's first zero at $f=1 / X$ and the high frequency components of $I(f)$.

Finally the spectrum $P_{s}(f)$ of the sampled signal $p[n]$ (corresponding to the detectors output level), based on (10), is given by (11).

$$
P_{s}(f) \approx T \cdot X \cdot e^{-j \pi \cdot f \cdot X} \cdot \sum_{-\infty}^{\infty}(-1)^{k} I\left(f-\frac{k}{X}\right) .
$$




\section{Resolution Beyond the Diffraction Limit}

Switching domain and reducing the problem to one dimension so that $I_{i}(f), H(f)$ and $I_{g}(f)$ are the spectrums of $i_{i}(u),|h(u)|^{2}$ and $i_{g}(u)$ respectively, it is immediate that $I_{i}(f)=H(f) I_{g}(f)$.

Assuming an $X$ small enough so (10) can be used, that most of the information in $I_{i}(f)$ is located at low spatial frequencies and that $H(f)$ is a good enough anti-aliasing LPF it is possible to simplify (11) down to (12) in the $f \in(-1 / 2,1 / 2)$ interval.

$$
P_{s}(f) \approx T \cdot X \cdot e^{-j \pi \cdot f \cdot X} \cdot H(f) \cdot I_{g}(f) .
$$

The recoverability of the diffraction free image from the detected one is reflected in (12). The resolution improvement arises from the possibility of using detectors which are smaller than the diffraction pattern.

\section{Reduction of the Number of Sen- sors: Decimation and Interpolation}

There is an important fact, already mentioned in the introduction, which is crucial in regard to the system complexity. Diffraction transforms point sources into blobs, and if small-enough detectors are used then information is spread among several of them. This allows a reduction in the number of detectors (decimation) thus reducing the system complexity. The value of the removed sensors can be interpolated. On the cons side a more restrictive (narrow banded) LPF $H(f)$ is required as will be shown.

Given the original signal $p[n]$ and its spectrum $P_{s}(f)$, the spectrum $P_{i}(f)$ of the signal resulting of applying a decimation factor $M$ followed by the interpolation process required to recover the decimated samples using an interpolation filter $H_{i}(f)$ is given by (13)

$$
P_{i}(f)=\frac{1}{M} H_{i}(f) \sum_{k=0}^{M-1} P_{s}\left(f-\frac{k}{M}\right) .
$$

It is clear that if $P_{s}(f)$ is to be recovered from $P_{i}(f)$ then the former has to be band limited to the interval $f \in(-1 / M, 1 / M)$. According to (12) $H(f)$ is the one that has to do the job of band limiting $I_{g}(f)$.

An important fact to remark is that whichever decimating factor $M$ is applied the system complexity (i.e. number of detectors) decays by a $1 / M^{2}$ factor due to the bidimensionality of sensor matrix. This implies that small decimation factors such as 2 or 3 produce a system complexity reduction of 4 and 9 times respectively.

\section{Proposed Method and Results}

The method that has been developed puts together all the ideas presented to this point. It uses the diffraction pattern produced by the diaphragm (assumed known and constant for the entire image) to create a spatial diversity (phase plane) strategy to be able to perform the detection in optimal conditions. It assumes that the blur generated by the diaphragm is perfectly reversible by just applying the inverse function (Fig. 8).

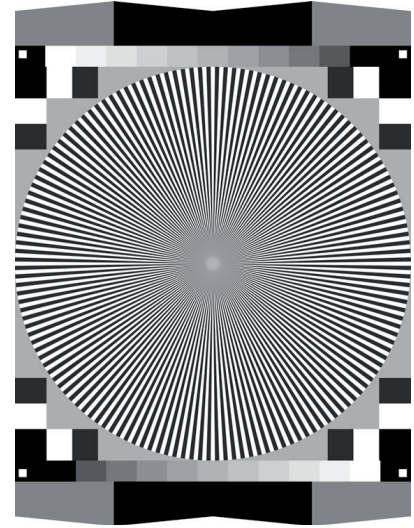

(a)

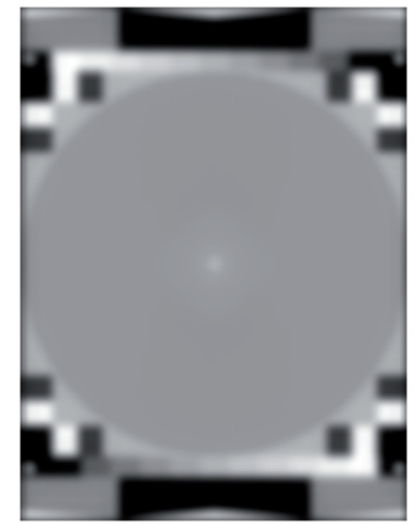

(b)

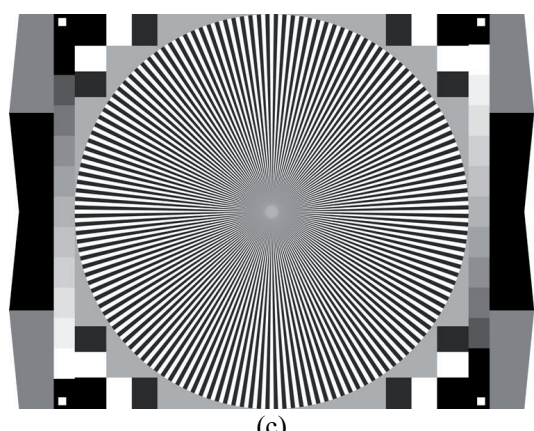

(c)

Fig. 8. (a) Original image. (b) Blurred image. (c) Recovered image.

Now we go a step forward and apply the decimation/interpolation process explained in section 6. Taking advantage of the fact that the diffraction pattern is a slow variating function (LPF) over the image (many detectors will be "sampling" that function rather than integrating it) we can interpolate new points in it thus increasing, artificially, the number of sensors and therefore improving the resolution of the final image. In order to compare the results, we decrease intentionally the number of detectors (pixels) once the original image (Fig. 8a) has been blurred by the diaphragm. In Fig. 9, a point source (a), which originally would be missed by the array, is spread through the sensors by diffraction (b). Once detected, the missing values are calculated through interpolation (c) and then the whole image is de-blurred (d) and presented.

In Fig. 10 and Fig. 11, an inverse problem -maintaining the resolution (number of pixels) of the final image and reducing the required detectors- is presented. The image is detected by a decimated array of sensors (Fig. 10a), then it is interpolated (Fig. 10b) and finally it is deblurred (Fig. 11 right). 


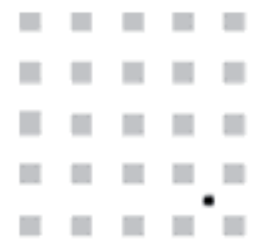

(a)

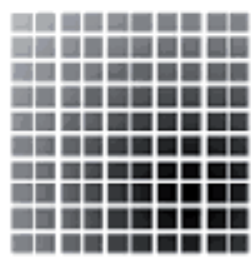

(c)

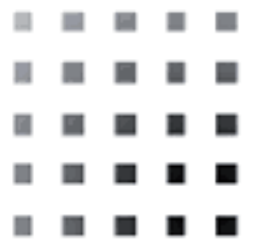

(b)

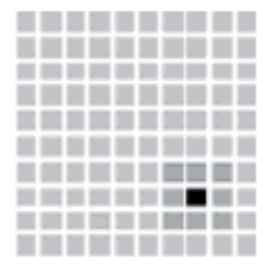

(d)

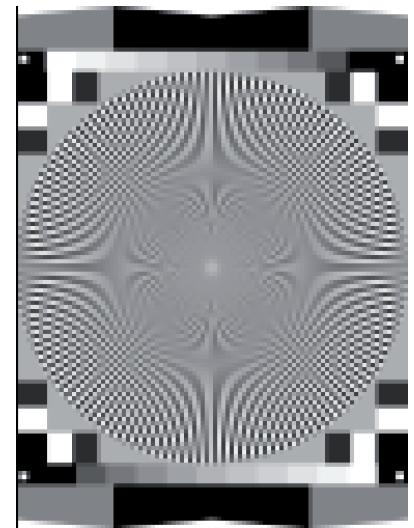

(b)

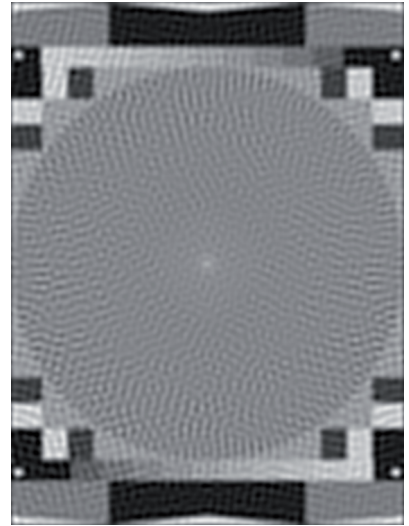

Fig. 9. The proposed method: (a) point (direction) to be detected, (b) blurred image over the detectors, (c) interpolated image and (d) final improved image.

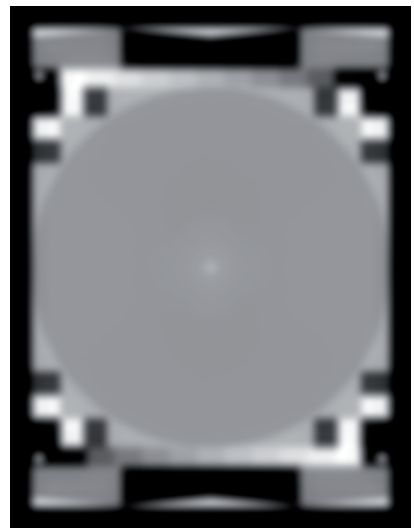

(b)
Fig. 10. Size comparison, assuming equal size for the detectors and for the final pixels, between the image captured by a decimated CCD (a) and the interpolated image (b).

The images obtained using different number of detectors are shown Fig. 11, proving that the shapes (high frequencies) are properly restored despite the reduced number of sensors. On the left column the result of using a classic CCD with certain amount of detectors is shown while the right column shows the result of using our method with the very same amount of detectors.
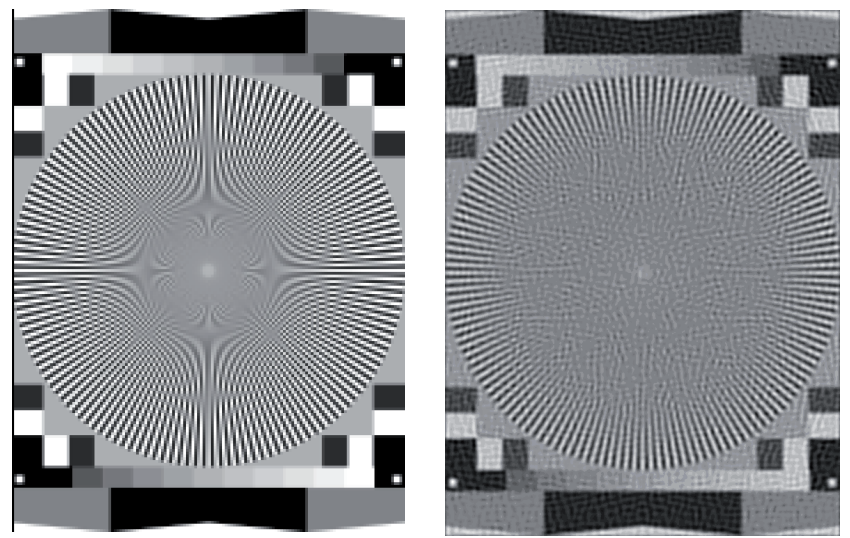

(a)
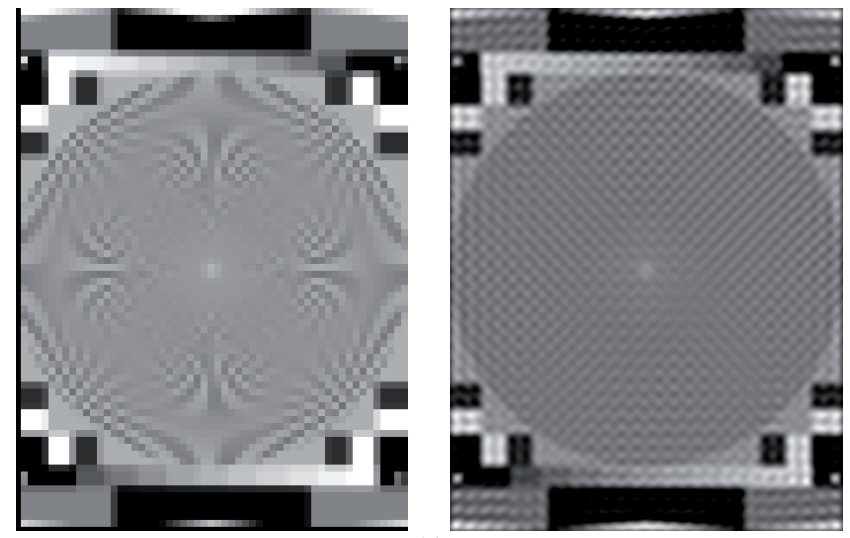

(c)

Fig. 11. The original image of $N \mathrm{x} N$ sensors shown in Fig. 8 treated with the proposed method. Image obtained with $N \mathrm{x} N / 16$ detectors (a), with $N \mathrm{x} N / 36$ (b) and $N \mathrm{x} N / 100$ (c).

\section{Conclusions}

Two main conclusions arise from this study: first, it is possible to get resolution beyond the diffraction limit (Fig. 4); second, the method proposed reduces significantly the number of sensors needed to achieve certain resolution. Note that a decimation factor $M$ implies a $M^{2}$ reduction in the number of sensors.

Last but not least, our method produces a more robust system since the interpolation applied recovers not only the decimated pixels, but also the damaged ones. This is possible because diffraction spreads out the information that in a CCD would be received by a single pixel across several ones.

\section{References}

[1] KOLB, H., FERNÁNDEZ, E., NELSON, R. Visual Acuity. WebVision. [Online]. Available at:

[2] ABRAMOWITZ, M. et al. Concepts in digital image technology. Optical Microscopy Primer. [Online]. Available at: http://webvision.med.utah.edu/KallSpatial.html. [July $3^{\text {rd }} 2009$ ]. 
http://micro.magnet.fsu.edu/primer/digitalimaging/concepts/conce pts.html. February $21^{\text {st }} 2005$ [September $25^{\text {th }} 2009$ ].

[3] SPRING, K. R., FELLERS, T. J., DAVIDSON, M. W. Introduction to Charge-Coupled Devices (CCDs). MicroscopyU. [Online]. Available:

http://www.microscopyu.com/articles/digitalimaging/ccdintro.html [September $28^{\text {th }} 2009$ ].

[4] STEWARD, E. G. Fourier Optics: An Introduction. $2^{\text {nd }}$ ed., Mineola: Dover, 2004.

[5] GOODMAN, J. W. Introduction to Fourier Optics. $3^{\text {rd }}$ ed., Englewood: Roberts \& Company, 2005.

[6] ERSOY, O. K. Diffraction, Fourier Optics and Imaging. Hoboken: Wiley, 2007.

\section{About Authors ...}

Juan C. LIZARRAGA was born in Bogotá, Colombia, in 1985. He received his M.Sc. in Telecommunication Engi- neering and M.Sc. in Radiocommunications from the Public University of Navarra, Spain, in 2009 and 2010 respectively. His research interests include lens antennas, antennae arrays and beam-forming networks.

Carlos DEL RIO was born in Reus, Spain. He received the $\mathrm{Ph}$. D. in Telecommunications (with honors) in the Public University of Navarra in 1996. Since 1993, he is associate professor in the Electronic and Electronic Engineering Department of the Public University of Navarra and the leader of the Antenna Research Group. His research interest include horn antenna design in general, satellite and terrestrial communications, study of periodic structures, Coherently Radiating Periodic Structures (CORPS), cooperative beam forming networks, meta-materials applied to antenna applications, development of numerical computation software based on mode matching and scattering matrix, antenna measurements, far-and near field measurement chambers, compact ranges, etc. 